\title{
Miranda
}

Revue pluridisciplinaire du monde anglophone /

Multidisciplinary peer-reviewed journal on the English-

speaking world

19 | 2019

Rethinking Laughter in Contemporary Anglophone Theatre

\section{François-René de Chateaubriand, Voyage en Amérique. Édition de Sébastien Baudoin.}

\section{Christine Dualé}

\section{(2) OpenEdition}

\section{Journals}

Édition électronique

URL : http://journals.openedition.org/miranda/21301

DOI : $10.4000 /$ miranda.21301

ISSN : 2108-6559

\section{Éditeur}

Université Toulouse - Jean Jaurès

Édition imprimée

Date de publication : 7 octobre 2019

\section{Référence électronique}

Christine Dualé, «François-René de Chateaubriand, Voyage en Amérique. Édition de Sébastien

Baudoin. », Miranda [En ligne], 19 | 2019, mis en ligne le 11 octobre 2019, consulté le 16 février 2021

URL : http://journals.openedition.org/miranda/21301 ; DOI : https://doi.org/10.4000/miranda.21301

Ce document a été généré automatiquement le 16 février 2021.

\section{(c) (i) (3)}

Miranda is licensed under a Creative Commons Attribution-NonCommercial-NoDerivatives 4.0 International License. 


\section{François-René de Chateaubriand, Voyage en Amérique. Édition de Sébastien Baudoin.}

Christine Dualé

RÉFÉRENCE

François-René de Chateaubriand, Voyage en Amérique. Édition de Sébastien Baudoin.

Paris, Gallimard, Collection Folio classique, 2019. 749 p, ISBN : 978-2-07-046710-5. 


\section{Chateaubriand Voyage en Amérique}

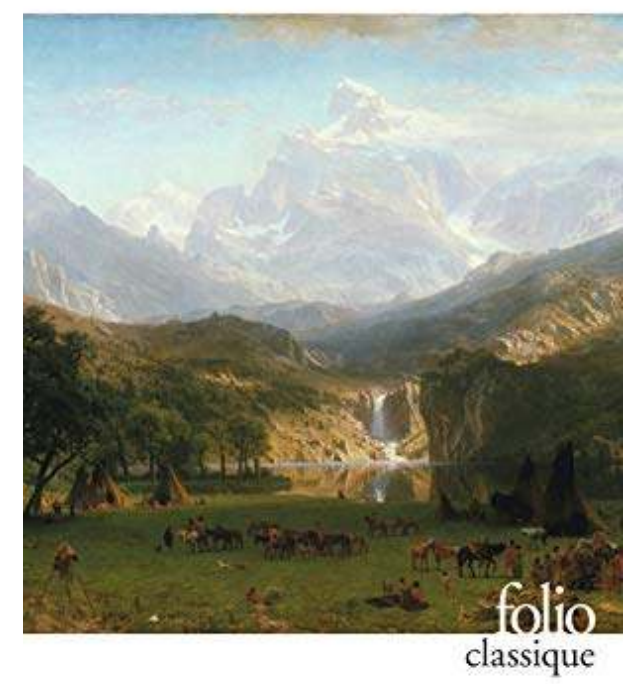

1 Dès la couverture de Voyage en Amérique par François-René de Chateaubriand édité par Sébastien Baudoin, le lecteur est transporté vers les contrées lointaines du Nouveau Monde où les Indiens, présentés ici au premier et second plans semblent pouvoir être déplacés au grès de la destinée manifeste de cette nouvelle nation évoluant inexorablement. Vue des Montagnes Rocheuses (1863) d'Albert Bierstadt, connu pour ses paysages grandioses de l'Ouest américain, est effectivement une entrée en matière très judicieuse lorsque l'on sait que l'artiste créa ce paysage idéal après son expédition pour trouver une route reliant le Mississippi au Pacifique et qu'il n'hésita pas à transformer certains détails afin de sublimer le paysage. Comme Bierstadt, Chateaubriand rêvait de découvrir un autre passage, celui du Nord-Ouest, et entreprit sa narration viatique trente-six ans après sa découverte du Nouveau Monde, «nourrissant sa nostalgie d'un passé revécu par l'écriture » (24) comme nous le précise l'éditeur. Chateaubriand révèle ici une nature grandiose et pittoresque teintée « d'émotion rousseauiste transcend[ant] souvent la réalité pour y voir germer les contours d'un idéal tendant vers l'infini métaphysique » (34). Dans la lignée de H. D. Thoreau et fasciné par la wilderness, c'est-àdire les grands espaces désertiques et sauvages, Chateaubriand dévoile rétrospectivement un décor sans équivalent dont les tableaux descriptifs contribuèrent à sa renommée de "peintre des paysages » (660). Sainte-Beuve le nomma d'ailleurs «l'Enchanteur » et loua « sa plume enchanteresse » (34).

2 La plume épique de ce « voyageur-poète » (35) est rééditée par Sébastien Baudoin aux Éditions Gallimard pour notre plus grand plaisir. La présente édition est très complète et agrémentée d'une chronologie détaillée (531-540), d'une notice sur l'épopée et la genèse du manuscrit (541-555), d'une bibliographie sélective sur Chateaubriand et la littérature de voyage, d'un répertoire des voyageurs cités par Chateaubriand (562-601) et d'une carte permettant de visualiser les itinéraires de Chateaubriand. La préface (7-51) très érudite et fouillée de Sébastien Baudoin est riche en références et notes explicatives dont les détails précis facilitent l'entrée dans le texte. Voyage en Amérique 
invite les lecteurs du vingt et unième siècle à la poésie, à la communion avec la nature et les grands espaces, alors que la réflexion de Chateaubriand se confond avec les questionnements philosophiques des Lumières. Lire ou relire Voyage en Amérique permet d'appréhender la nature vue par un homme du XVIII ${ }^{e}$ siècle, de la mettre en perspective avec la nature du XXI siècle et d'en saisir l'extrême fragilité. Comme le remarque Sébastien Baudouin, la nature décrite par Chateaubriand « est à la démesure de ce qu'il attendait » (41), lui qui s'interrogeait déjà sur le futur de l'Amérique et les découvertes des explorateurs: "Est-il bien que les communications entre les hommes soient devenues aussi faciles? Les nations ne conserveraient-elles pas mieux leur caractère en s'ignorant les unes les autres, en gardant une fidélité religieuse aux habitudes de leurs pères? » (111) Il s'interroge aussi rétrospectivement sur la grande hétérogénéité de la population et sa possible homogénéité: "[C]elui-là catholique, paresseux et superbe, celui-là luthérien, laboureur et sans esclaves ; celui-là anglican et planteur avec des nègres ; celui-là puritain et négociant ; combien faudra-t-il de siècles pour rendre ces éléments homogènes! » (522).

du voyage en Orient (1806-1807) Chateaubriand affirme qu'il était préférable de voir la Grèce avec les yeux d'Homère et de demeurer un voyageur immobile afin d'éviter toutes désillusions, son grand voyage en Amérique l'entraîne toutefois vers des contrées "sources de révélations esthétiques et métaphysiques » (36). Entre déception et illusion, expérience extatique et émotion rousseauiste, Voyage en Amérique " reprend la tradition des grands voyageurs » (21) dont il se réclame et auxquels il s'identifie : «je viens me ranger dans la foule des voyageurs obscurs qui n'ont vu que ce que tout le monde a vu, qui n'ont fait faire aucun progrès aux sciences [...], mais je me présente comme le dernier historien des peuples de la terre de Colomb, de ces peuples dont la race ne tardera pas à disparaître; je viens dire quelques mots sur les destinées futures de l'Amérique, sur ces autres peuples héritiers des infortunés Indiens : je n'ai d'autre prétention que d'exprimer des regrets et des espérances » (111). Chateaubriand veut écrire dans la lignée d'Hérodote, qu'il présente comme le "père de l'histoire » (606). Comme lui, il se veut le « garant » de l'Histoire.

4 Le voyage en lui-même fut pour Chateaubriand un moyen de fuir la tutelle familiale et de donner corps à ses rêves exaltés de «jeune homme désabusé et sans avenir, chassé par les désordres de l'Histoire, réfugié dans la folie de ses rêves d'absolu (9) »; un jeune homme en proie à " un imaginaire enflammé en quête d'exotisme et d'ailleurs » (9) qui tente de vivre dans ce Nouveau Monde une expérience à la hauteur de son imaginaire et qui va lui fournir l'inspiration de toute son œuvre.

5 Le lecteur retrouve ainsi les grands courants de la littérature française mais aussi américaine dans la description des paysages notamment. Les échos à Thoreau, les recours à Bartram ou encore Gilpin sont fréquents. Le pittoresque, le sublime, le transcendantalisme, la wilderness, l'expérience extatique sont convoqués pour évoquer ce Nouveau Monde, « nous mystifier et nous éblouir » (37). Les références régulières à Mémoire d'outre-tombe, Génie du christianisme, Atala, Essai sur les révolutions, Les Natchez et aux ouvrages de William Bartram (Voyages) ou de H.D. Thoreau (Un Yankee au Canada; Walden) dans les notes de l'éditeur viennent étayer les descriptions de l'auteur et ajoutent à la précision du vaste et riche panorama historique et littéraire proposé par Sébastien Baudoin. Ainsi, le principe d'écriture de Chateaubriand transparaît à travers les références et les échos de l'éditeur aux autres textes de Chateaubriand, lui qui concevait ses récits comme une mosaïque « [en] recycl[ant] ses propres textes » (542) et 
en empruntant à d'autres auteurs-voyageurs. Voyage en Amérique fonctionne comme un réservoir qui "servira à abreuver la grande œuvre, somme de sa vie, les Mémoires » (547). Chateaubriand prolongea ainsi sa réflexion dans Les Natchez, Atala, le Génie du christianisme, ou encore les Mémoires d'outre-tombe. Dans la partie Notice (541-555), placée après le récit et les notes de Chateaubriand, Sébastien Baudoin propose une synthèse supplémentaire très éclairante sur "L'épopée du manuscrit », sa genèse, sa conception et sa réception.

6 Dans la lignée de la tradition des récits viatiques, Voyage en Amérique est organisé autour d'une structure tripartite (Itinéraire, Histoire naturelle, Moeurs des Sauvages), se clôt sur une réflexion sur les États-Unis puis sur les Républiques espagnoles et la fin du voyage. L'ouvrage suit " un principe de montage », comme l'indique Sébastien Baudoin en référence à Philippe Antoine dans Récits de voyage de Chateaubriand, contribution à l'étude d'un genre (Paris, Honoré Champion 1997, 24). Voyage en Amérique emprunte en définitive à plusieurs modèles de la littérature de voyage car Chateaubriand n'hésite pas à reprendre abondamment le texte de Bartram notamment pour retracer son soidisant itinéraire en Floride dans «Description de quelques sites dans l'intérieur des Florides » (179-191) alors qu'il ne s'y est jamais rendu. Carver, Charlevoix, Bartram sont des sources inépuisables pour Chateaubriand et les critiques mirent en doute la véracité de ses propos. Chateaubriand est tour à tour historien, géographe, naturaliste, archéologue, ethnologue, et poète. Très inspiré, ses descriptions cherchent à « éblouir le lecteur » (685) et il a recours à « de nombreux effets de théâtralisation » (668) tout en exprimant un « idéal rousseauiste [...] celui de retrouver l'homme primitif, vivant selon les lois de la nature, dans sa pureté originelle [...]» (669), comme l'explique avec précision Sébastien Baudoin.

7 Le spectacle de la nature hybride avec laquelle Chateaubriand est en contact, entre espaces sauvages (wilderness) et terres détruites par les colons, est loin de le laisser indifférent. Il ne manque d'ailleurs pas de remarquer: «les puissances civilisées se partagent sans façon, en Amérique, des terres qui ne leur appartiennent pas » (133) et d'ajouter plus loin: "les Européens ont remplacé ces bienfaits de la nature par les productions de l'art : les Sauvages ont disparu » (149). Les descriptions de la nature lui permettent aussi de mettre en scène ses émotions et d'évoquer la présence de Dieu, génie créateur et ordonnateur de la nature. Il devient naturaliste dans la partie intitulée Histoire naturelle. Dans Mours des Sauvages il rapproche les mœurs des Indiens de celles des Anciens "rêvant d'une origine commune de simplicité de mœurs, de vertu, qui renverrait à l'enfance de l'âge humain, non encore perverti par la société moderne" (699). Après des élans lyriques lorsqu'il évoque les vastes espaces américains, Chateaubriand décrit avec objectivité «les tribus errantes du Nouveau Monde » (324), leurs mœurs et coutumes et « peint ce qui fut beaucoup plus que ce qui est » (324). En faisant état des territoires spoliés des Indiens, il adopte la posture de l'historien, "celle du dernier témoin" (720) qui, comme le remarque Sébastien Baudoin, "confère à son discours un intérêt particulier " (720). Dans la conclusion Chateaubriand souligne le développement effréné et inexorable de l'Amérique, son "esprit mercantile» (348) ainsi que le bien-être matériel des Américains tout en s'interrogeant sur « les prodiges de la liberté » (347). Il oppose les principes de liberté des Amériques espagnoles à ceux des États-Unis et "expose avec franchise les difficultés qui [...] entrav[èrent] la liberté des républiques espagnoles » (360). Puis les 
circonstances de l'Histoire et sa fibre royaliste poussent Chateaubriand à revenir en France. Fin du voyage dresse ainsi le bilan de ses « destinées vagabondes » (367).

On retiendra le travail remarquable d'érudition et de contextualisation de Sébastien Baudoin tant dans sa préface que dans les notes riches en références et explications qui permettent de replacer ce voyage en Amérique dans le contexte des voyages d'explorateurs et d'identifier les sources de Chateaubriand. Le «Répertoire des voyageurs» constitué par Sébastien Baudoin, qui regroupe les noms d'auteursvoyageurs cités par l'auteur, est aussi d'une grande précision et vient compléter une préface et Notice très détaillées où les rappels historiques, littéraires et bibliographiques sont nombreux. Les multiples renvois aux autres ouvrages de Chateaubriand sont particulièrement judicieux pour pénétrer l'ensemble de son œuvre et témoignent de la connaissance très pointue de l'écriture de Chateaubriand par Sébastien Baudoin.

Lire Voyage en Amérique de Chateaubriand, édité par Sébastien Baudoin, permet de découvrir ou redécouvrir un texte moins connu qu'Atala ou encore Mémoires d'outretombe, d'exhumer un monde oublié, l'Amérique de la fin du XVIII siècle « désormais bel et bien morte » (51), dont la fascination demeure et de réfléchir à un auteur-voyageur finalement en avance sur son époque, nostalgique d'une grandeur passée et rempli d'espoir en ce Nouveau Monde où certes « le genre humain recommence » (338) mais au prix d'une nature et de nations indiennes spoliées. Comme l'édition de Sébastien Baudoin le démontre, «le voyage en Amérique demeure inscrit en lettres de noblesse dans la littérature française par le récit de Chateaubriand, notamment par le relais des Mémoires d'outre-tombe, qui en ont repris les plus belles pages pour les livrer à la postérité » (554).

\section{INDEX}

Mots-clés : Amérique, Destinée Manifeste, explorateurs, littérature/ récit de voyage, Nature, Nouveau Monde, Passage du Nord-Ouest, grands espaces, transcendantalisme, la wilderness

Keywords : Travel account/ literature, America, explorers, Manifest Destiny, Nature, the New World, North-West Passage, open spaces, transcendentalism, wilderness

\section{AUTEURS}

\section{CHRISTINE DUALÉ}

Professeur - Culture noire américaine

Université Jean Monnet - Saint Etienne

christine.duale@univ-st-etienne.fr 